\title{
Análisis de comportamientos relacionados con el uso/abuso de Internet, teléfono móvil, compras y juego en estudiantes universitarios
}

\section{Analysis of behavior related to use of the Internet, mobile telephones, compulsive shopping and gambling among university students}

Rosario Ruiz-Olivares*; Valentina Lucena*;

M. José PINO*; JAVIER HERRUZO*
* Facultad de Ciencias de la Educación, Universidad de Córdoba

** Centro Renacer, Córdoba.
Enviar correspondencia a

Javier Herruzo jherruzo@uco.es

Facultad de Ciencias de la Educación. Departamento de Psicología.

Calle San Alberto Magno, s/n. 14071 Córdoba (España)
RESUMEN

El objetivo de este trabajo de investigación es conocer los hábitos relacionados con posibles conductas adictivas (juego patológico, Internet, compras, uso del teléfono móvil, etc.) que pueden presentar los jóvenes estudiantes de la Universidad de Córdoba (España), y relacionar dichos comportamientos con variables como la edad, el sexo, el curso y las macroáreas de conocimiento (letras y ciencias). Con un diseño expostfacto de grupo único (Montero y León, 2007), se elaboró un cuestionario de datos sociodemográficos en el que se incluia el Test de adicción a las compras, el Test de adicción a Internet de Echeburúa (2003), el Cuestionario Breve de Juego Patológico de Fernández-Montalvo y Echeburúa (1997) y algunos ítems sobre el uso del teléfono móvil. Participaron 1011 estudiantes, $42,7 \%$ hombres y $57,3 \%$ mujeres, con un rango de edad entre los 18 y 29 años. Se dan diferencias estadisticamente significativas entre la puntuación media obtenida en los cuestionarios y variables como la edad, el sexo, el curso o la macroárea de conocimiento. Parece que ser mujer es un factor protector para la adicción a Internet y al Juego, ser de ciencias es un factor de riesgo para una posible adicción al Juego, ser de letras y tener más edad son factores de riesgo ante una posible adicción a las Compras. En conclusión, los estudiantes encuestados realizan un uso moderado de conductas como navegar por Internet, el juego, las compras y el teléfono móvil, siendo un grupo muy reducido de jóvenes los que sí se encuentran cercanos a tener un problema de adicción con este tipo de comportamientos.

Palabras clave: adicción, móvil, Internet, compras, juego, universitarios.
ABSTRACT

The aim of this study is to obtain knowledge about habits related to addictive behaviour (pathological gambling, Internet, compulsive shopping, use of mobile telephones, etc.) that may be displayed by young students at the University of Cordoba (Spain), and to relate this behaviour with variables such as age, sex, course year, macro-field of study (arts/sciences) and the consumption of substances such as alcohol, tobacco, cannabis and cocaine. Using an ex-post facto single-group design (Montero \&t León, 2007), we applied a questionnaire especially designed to gather socio-demographic information on substance use and behavioural patterns related to "non-substance" addictions, which included the Shopping Addiction Test, Echeburúa's Internet Addiction Test (2003) and Fernández-Montalvo and Echeburúa's Short Pathological Gambling Questionnaire (1997). A total of 1,011 students participated in the study (42.7\% males and 57.3\% females), with an age range of 18 to 29. Significant differences were found between mean score on the questionnaires and variables such as age, sex, field of studies and course year. It would seem that being female is a protective factor for Internet and gambling addiction, being a sciences student is a risk factor for gambling addiction, and being older and being an arts student are risk factors for shopping addiction. In conclusion, it can be stated that the students surveyed showed moderate incidence of behaviours such as Internet browsing, gambling, shopping and mobile phone use, whilst a very small group are close to having an addiction problem with such behaviours.

Key Words: addiction, mobile telephone, Internet, shopping, gambling, university students. 


\section{INTRODUCCIÓN}

E nuestra sociedad, conductas como comprar, jugar, trabajar, practicar sexo, etc. son conductas socialmente aceptadas y tienen la característica común de proveer un estado de gratificación inmediata (Marlatt y Gordon, 1985). Cuando la relación que la persona mantiene con ellas comienza a ser problemática (porque pasa excesivo tiempo realizándolas, porque deja de hacer otras cosas de más relevancia, porque el tiempo que no está realizándolas lo está deseando, etc.), se considera que la persona padece una adicción conductual (Alonso-Fernández, 2003; Echeburúa, 1999; González Duro, 2005; Holden, 2001; Lemon, 2002). El progreso social y el avance de las Tecnologías de la Información y la Comunicación (TICs) promueven nuevos patrones de comportamientos susceptibles de promover un abuso y cierta dependencia, como es navegar por Internet y el uso del teléfono móvil (Sánchez-Carbonell, Beranuy, Castellana, Chamarro y Oberst, 2008). Conocer y analizar este tipo de comportamientos parece lógico, ya que pueden tener síntomas y consecuencias, al menos en parte, de similares características a las que presentan las personas adictas a alguna sustancia. La población de estudiantes universitarios merece especial atención, pues junto con la adolescencia, son los que presentan mayor sensibilidad a realizar dichas conductas, ya sea por las condiciones en las que viven (fuera del hogar), el escaso control que tienen sobre el dinero, las responsabilidades de la vida diaria, y/o la necesidad que tienen de contactar con los amigos de otros lugares (Castellana, Sánchez-Carbonell, Graner y Beranuy, 2007; Sánchez-Carbonell et al., 2008).

La conducta de navegar por Internet se define como el uso de aplicaciones comunicativas y sincrónicas como chats, juegos en línea, redes sociales, etc. (Viñas, Juan, Villar, Caparros, Pérez y Cornella, 2002). Además de ser en sí misma una conducta reforzante con tendencia a crear dependencia (Young, 1996), el uso de Internet provee a la persona de otras conductas reforzantes que también tienen la capacidad de producir dependencia, como son las relacionadas con el sexo, el juego de apuestas o las compras (Viñas, et al., 2002). Según algunos autores (Echeburúa, 2003; Sánchez-Carbonell, et al., 2008) se puede hablar de dependencia psicológica a Internet cuando aparece preocupación por conectarse cuando no se está conectado; se limitan las formas de diversión; se reducen las relaciones sociales y la actividad física; el estado de ánimo se ve alterado; se permanece muchas horas conectado, siendo la persona incapaz de interrumpir la conexión; aumenta el aislamiento social y se deterioran las relaciones más cercanas (Castellana, et al., 2007; García, Terol, Nieto, Lledó, Sánchez, Martín-Aragón y Sitges, 2008; Muñoz-Rivas, Navarro y Ortega, 2003; Sánchez-Carbonell et al., 2008).

En un estudio realizado por Muñoz-Rivas, et al. (2003) con universitarios españoles de la Universidad Complutense de Madrid, se encontró que el 57\% de los estudiantes eran usuarios de la red, de los que el 52,7\% utilizaban la red de 0 a 2 horas y el $27,6 \%$ de 3 a 6 horas, y donde se observaba que el recurso más utilizado y en el que se invertían más horas era el acceso a paginas webs (77\%), el 37,4\% para el acceso a líneas de chats y el $16,8 \%$ a juegos en red. El porcentaje de hombres era casi siempre superior al de mujeres, el 17\% de los jóvenes afirmaban que el uso de Internet les había provocado consecuencias negativas en su vida diaria como descuidar sus obligaciones $(10,9 \%)$, problemas con la familia $(3,6 \%)$, disminución del rendimiento escolar $(2,4 \%)$ e incluso el $0,9 \%$ declaró que habían perdido amigos por el tiempo empleado en la red.

En otro estudio se describe la relación entre la frecuencia de uso de Internet y variables psicosociales entre estudiantes universitarios españoles (Garcia, et al., 2008). En este trabajo, se encontró que un $73,9 \%$ se conectaba semanalmente con un tiempo medio de 84.81 minutos, siendo los alumnos de Psicología los que mostraban mayor porcentaje de uso $(4,8 \%)$ "varias veces al dia", y Podología y Relaciones Laborales los que menos frecuencia presentaban, siendo inferior a los límites de conexión propuestos en el estudio. De los estudiantes que se conectaban, el 80,5\% estaban más de media hora y hasta dos horas. Se llega a la conclusión de que no existía una presencia de comportamientos de abuso de Internet en los estudiantes universitarios, pero si un cambio en los estilos de vida y comportamientos sociales actuando como refuerzo; el grupo que más usaba Internet parecia que ampliaba sus relaciones sociales en este medio y le servía para sentirse mejor o evitar problemas o emociones displacenteras, provocando cambios en el tiempo dedicado a otras actividades.

La posible adicción por el uso excesivo del teléfono móvil se define como la incapacidad para controlar o interrumpir su uso bloqueando las llamadas o la cuota, desconectando el sonido o apagando el terminal (Muñoz-Rivas y Agustín, 2005; Sánchez-Carbonell, et al., 2008). Se dice posible, pues no todos están de acuerdo en afirmar que el uso excesivo del teléfono móvil puede crear dependencia (SánchezCarbonell, et al., 2008). Sin embargo, el teléfono móvil presenta una característica especial que lo hace susceptible de producir ciertos comportamientos abusivos, como son la sociabilidad, autoconfianza, diversión, estatus social, movilidad, acceso permanente, identidad, conciliación familiar e individualización de bienes (Dimmick, Kline y Strafford, 1994; Höflich y Rössler, 2002; Leung y Wei, 2000; 0’Keefe y Sulanowski, 1995). Este uso inapropiado del teléfono móvil conlleva una serie de efectos negativos como el gasto excesivo (Criado, 2005; Protégeles, 2005), riesgos en la conducción (Adès y Lejoyeux, 2003; MuñozRivas y Agustín, 2005), efectos secundarios en la salud como los derivados del sueño y problemas en el ámbito laboral, social, familiar y escolar (Muñoz-Rivas y Agustín, 2005). Según estudios realizados con adolescentes, pueden llegar a priorizar la comunicación mediada por el móvil alterando sus relaciones sociales, (Bianchi y Phillips, 2005; Kamibeppu y Sugiura, 2005) e incluso, llegando a no saber manejarse en las relaciones cara a cara (Criado, 2005).

Las personas que presentan una adicción conductual al juego son aquellas que no pueden resistirse y se dedican a esta actividad de forma creciente y descontrolada, llegando incluso a entrar en conflicto con sus objetivos personales 
y familiares, impidiendo en la mayoría de los casos el desarrollo de una vida normal (Atienza, 2006; Becoña, 1993, 1996; Echeburúa y De Corral, 2008; Secades y Villa, 1998). En un estudio de Lesieur y Rosenthal (1991), ya se ponía en evidencia que la prevalencia de juego problemático entre estudiantes universitarios era de cuatro a ocho veces mayor que en adultos. $Y$ en otros estudios realizados con estudiantes de Secundaría también se daban altas prevalencias, un 2,4\% de probables jugadores patológicos en La Coruña (Becoña, 1996), y en otro estudio (Becoña, Míguez y Vázquez, 2001) se encontró que entre los estudiantes de Secundaria de Galicia un $8,2 \%$ se consideraban jugadores en riesgo y un $5,6 \%$ jugadores con problemas, siendo mayor la prevalencia en hombres que en mujeres de 7:1. En Andalucia, se volvían a repetir los resultados, destacando Huelva con un 2,7\% de los estudiantes (Arbinaga, 1996a, b). Arbinaga (2000) encontró, en un estudio realizado con estudiantes de 8-17 años, que el $11,5 \%$ de los estudiantes presentaban problemas leves con el juego y el $5,4 \%$ se podria clasificar como jugador patológico, presentando un fuerte incremento en las mujeres dentro del grupo de los que presentaban problemas leves.

Consumir objetos, comprar, una actividad rutinaria de la vida diaria para la mayoría de la gente, también se puede convertir en un comportamiento adictivo, si se realiza para otros fines distintos a cubrir una necesidad (Echeburúa, 2003). Las personas con este tipo de comportamientos, se muestran incapaces de controlar sus vidas produciéndose consecuencias graves, ya que suelen consumir cosas no planificadas que exceden normalmente sus posibilidades económicas. La mayoría de estas personas tienen alrededor de 30 años, pero es en la juventud y adolescencia donde se inicia el problema, alrededor de los 18-20 años (Becoña, 2003). En la actualidad, apenas existen datos en la literatura científica relacionados con los jóvenes universitarios y la posible conducta adictiva de comprar.

Por todo ello, parece interesante analizar la prevalencia de los jóvenes universitarios de Córdoba en relación a conductas relacionadas con las TICs (Internet, teléfono móvil), el juego y las compras. Conocer si existe riesgo de tener un problema de adicción conductual con alguno de los comportamientos anteriormente mencionados, es importante para poder llevar a cabo actividades y estrategias adecuadas de prevención. El objetivo de este trabajo de investigación es conocer los hábitos relacionados con posibles adicciones conductuales (juego patológico, Internet, compras, uso del teléfono móvil) que pueden presentar los jóvenes estudiantes de la Universidad de Córdoba y relacionar dicha prevalencia con variables como la edad, el sexo, el curso y la macroárea de conocimiento.

\section{MÉTODO}

\section{Diseño}

El estudio se ha realizado mediante un diseño expostfacto prospectivo de grupo único (Montero y León, 2007).
La población objetivo eran 15.000 alumnos de la Universidad de Córdoba (UCO). El proyecto se planteó con el visto bueno del Vicerrectorado de Estudiantes de la UCO que solicitó mediante un escrito formal la colaboración de los decanos y administradores de todos los centros. Para la predeterminación del tamaño de la muestra, se partió de la siguiente simulación realizada con el paquete estadistico Epilnfo 2000, que para un 1\% de frecuencia esperada y un margen de error del 0,01 , se obtuvo que como mínimo se debía recoger la información de 1000 sujetos.

\section{Características de la muestra}

En el estudio han participado 1011 estudiantes universitarios de los diferentes centros que componen la Universidad de Córdoba, siendo el 42\% (428) hombres y 56\% (575) mujeres; el 59,3\% (600) estudian una carrera de la macroárea de ciencias y el 40,7\% (411) una carrera de la macroárea de humanidades, ciencias sociales y jurídicas. Por edades y en función de los rangos que se establecian en el cuestionario para recoger el dato de la edad, la muestra se distribuye en un 53\% (536) de 18-20 años, un 30,6\% (309) de 21-23 años, un 11,1\% (112) de 24-26 años, un 2,6\% (26) de $27-29$ años y 2,5\% (25) más de 29 años.

Dentro de cada uno de los centros, el muestreo se realizó por conglomerados tomando el aula como unidad muestral. Las aulas participantes fueron seleccionadas al azar tomando en consideración la disponibilidad de los profesores y de los alumnos dependiendo del calendario del programa docente.

\section{Instrumentos y variables}

Las principales fuentes para elaborar el cuestionario fueron la encuesta sobre drogas del Plan Nacional sobre Drogas y del Plan Andaluz sobre Drogas, y el cuestionario utilizado en el trabajo de Arenas, Carmona, Rodríguez y Tatnell (2002) con población universitaria de la ciudad de Córdoba que tenía como objetivo describir los patrones de consumo de las diferentes sustancias en función de variables como la edad, sexo, estudios universitarios, religión, política, características familiares y nivel socioeconómico dirigido hacia futuras campañas de prevención en el ámbito universitario. Para valorar el uso/abuso de las compras, se utilizó el Test de adicción a las compras de Echeburúa (2003), formado por cinco ítems, con dos alternativas de respuesta "si" o "no", considerando que la persona tiene un problema de adicción a las compras si responde afirmativamente a dos o más ítems; para valorar el comportamiento relacionado con el juego patológico, se utilizó el Cuestionario Breve de Juego Patológico (CBJP) ( $\alpha$ de Cronbach $=0,94$ ) de Fernández-Montalvo, Echeburúa y Báez (1995), formado por 4 ítems, con dos alternativas de respuesta "si" o "no", considerando que la persona tiene un problema con el juego si responde afirmativamente a dos o más ítems; para valorar la posible adicción al uso de Internet, se ha utilizado el Test de adicción a Internet (Echeburúa, 2003), formado por 9 ítems, con dos alternativas de respuesta 
"si" o "no", con una escala de puntuación dependiendo del número de respuestas afirmativas (Echeburúa, 2003, pág. 70). Así, de 0-3 respuestas afirmativas se considera que la persona no tiene problemas con el uso de Internet, de 4-6 respuestas afirmativas se considera que la persona está en riesgo de tener una dependencia y de 7-9 respuestas afirmativas es probable que la persona presente un problema de adicción a Internet. Para valorar el uso del teléfono móvil, se incluyeron una serie de items que hacian referencia al tiempo en horas que pasaban hablando o al número de mensajes que mandaban al dia los estudiantes (Bianchi y Phillips, 2005) ( $\alpha$ de Cronbach $=0,93$ ). Así, el cuestionario quedó dividido en un bloque de datos sociodemográficos y otro donde se obtenía información acerca de patrones de conducta relacionados con el uso de Internet, las compras, el teléfono móvil y el juego.

\section{Procedimiento}

La recogida de datos se realizó durante las dos primeras semanas de clase del curso 2007-2008. El profesorado previamente informado, dejó media hora de su clase para que los alumnos contestaran el cuestionario. En el apartado de instrucciones del mismo se especificaba que era una encuesta para conocer una serie de comportamientos en población universitaria, cuyo objetivo era la realización de una serie de actuaciones de prevención y tratamiento ante los problemas que podian derivarse de estas conductas. Por último, se resaltaba la importancia de su participación, informando a su vez que el tratamiento de los datos sería estadístico y anónimo

\section{Análisis de datos}

Con la información proporcionada por los cuestionarios se elaboró una base de datos en SPSS 12.0. El estudio estadístico descriptivo para el uso del teléfono móvil se llevó a cabo mediante análisis inferenciales que permitieron determinar relaciones entre variables cualitativas por medio de tablas de contingencia y contrastes Chi Cuadrado de Pearson. Se realizaron Análisis de Varianza (ANOVA) entre las puntuaciones medias de los cuestionarios (Adicción a Internet, Juego patológico y Adicción a las compras) y variables sociodemográficas como la edad, el sexo, el curso, etc. Por último, para cada cuestionario, se realizó una Regresión Logística binaria para indagar cómo actuaban las variables sociodemográficas anteriormente citadas (riesgo 0 protección).

\section{RESULTADOS}

\section{Patrón conductual de la muestra}

La muestra de jóvenes universitarios en relación a una posible adicción a las compras queda dividida en un $84 \%$ (831) de jóvenes que respondieron una o ninguna respuesta afirmativa del Test de adicción a las compras de Echeburúa (2003), y un 16\% (158) que respondió a dos o más ítems afirmativamente. Es decir, que el $16 \%$ de los alumnos universitarios tienen un posible problema de adicción a las compras.

En relación a la posible adicción a Internet, valorada con el Test de adicción a Internet de Echeburúa (2003), se puede decir que el 94,7\% (923) de los jóvenes que contestaron de $0-3$ respuestas afirmativas, tienen un uso normal de Internet; el 4,5\% (44) que respondió de 4-6 respuestas afirmativas presentan riesgo de tener una posible adicción a navegar por la red; y el 0,7\% (7) de los jóvenes que respondió de $7-9$ respuestas afirmativas tienen un posible problema de adicción a Internet.

En cuanto a la posible adicción al juego, valorada con el Cuestionario Breve de Juego Patológico de FernándezMontalvo y Echeburúa (1997), el 98,7\% (965) de los jóvenes que respondió afirmativamente a uno o ningún item no presentan una posible adicción al juego, frente al 1,3\% (13) de los jóvenes que respondió a dos o más ítems afirmativamente presentando una posible adicción al juego.

En cuanto al uso del teléfono móvil, el 32,6\% (326) de los jóvenes universitarios reconocen fracasar a la hora de reducir el gasto de móvil, el 22,6\% (223) pasa más de una hora al día hablando por el teléfono móvil, el 3\% (30) pasa entre 3 y 5 horas, el 75,3\% (746) manda menos de cinco mensajes de texto al dia, el 17,1\% (169) manda de seis a diez al día y el 7,6\% (75) manda más de diez.

\section{Diferencias en función del sexo}

Teniendo en cuenta las puntuaciones medias en cada uno de los cuestionarios en función de la variable sexo, se dan diferencias estadisticamente significativas según el Análisis de Varianza con una $F(1,965)=18,146 ; p<0,05$, para el cuestionario de Adicción a Internet; una $F(1,968)=35,395$; $\mathrm{p}<0,05$, para el cuestionario de Adicción al Juego; y una $F(1,979)=19,329 ; p<0,05$, para el cuestionario de Adicción a las Compras.

Tabla 1. Análisis de Varianza entre la variable sexo, y el Test de adicción a las compras, el Test de adicción a Internet (Echeburúa, 2003) y el Cuestionario Breve de Juego patológico (Fernández-Montalvo y Echeburúa, 1997).

\begin{tabular}{|l|c|c|c|c|}
\hline CUESTIONARIOS & SEXO & MEDIA & $\mathbf{F}$ & $\mathbf{p}$ \\
\hline \multirow{2}{*}{ INTERNET } & Hombres (421) & 1,1045 & \multirow{2}{*}{18,146} & \multirow{2}{*}{0,001} \\
\cline { 2 - 3 } & Mujeres (546) & 0,6813 & & \\
\hline \multirow{2}{*}{ JUEGO } & Hombres (414) & 0,1787 & \multirow{2}{*}{35,395} & \multirow{2}{*}{0,001} \\
\cline { 2 - 3 } & Mujeres (556) & 0,0378 & \multirow{2}{*}{19,329} & \multirow{2}{*}{0,001} \\
\hline \multirow{2}{*}{ COMPRAS } & Hombres (422) & 0,5213 & \\
\cline { 2 - 3 } & Mujers (559) & 0,7764 & \multicolumn{2}{|c}{} \\
\hline
\end{tabular}

En referencia al uso del móvil, en general las mujeres mandan más cantidad de mensajes de texto al día que los hombres. Concretamente, el 79,2\% (331) de los hombres mandan menos de cinco mensajes de texto al día frente al 
$72,2 \%$ (408) de las mujeres; el 13,9\% (58) de los hombres mandan de seis a diez mensajes frente al 19,6\% (111) de las mujeres y el $6,7 \%(28)$ de los hombres mandan más de diez frente al $8,2 \%(46)$ de mujeres $(p<.05)$. Además las mujeres reconocen un mayor fracaso a la hora de reducir el gasto de móvil con un 37\% (210) frente a un 27\% (113) de hombres que lo reconocen $(p<.05)$. Las mujeres también parece que son las que más tiempo pasan al día hablando por el móvil, ya que el 25,5\% (144) de mujeres habla más de una hora al día frente al 18\% (75) de los hombres que lo hacen $(p<.05)$.

\section{Diferencias en función de la edad:}

Relacionando las puntuaciones medias en cada uno de los cuestionarios en función de la variable edad, se dan diferencias estadisticamente significativas según el Análisis de Varianza en el cuestionario de Adicción al Juego con una $F(5,970)=2,997 ; p<0,05 ; y$ en el cuestionario de Adicción a las Compras con una $F(5,981)=3,957 ; p<0,05$. En la adicción al juego, destacan los rangos de edad 21-23, 24-26 y los mayores de 29 años por ser los que mayor puntuación media obtienen. En el caso del Cuestionario de Adicción a las Compras, destacan los rangos de edad 18-20, 21-23 y los mayores de 29. No se dan diferencias significativas en el cuestionario de Adicción a Internet.

Tabla 2. Análisis de Varianza entre la variable edad y, el Test de adicción a las compras, el Test de adicción a Internet (Echeburúa, 2003) y el Cuestionario Breve de Juego patológico (Fernández-Montalvo y Echeburúa, 1997).

\begin{tabular}{|c|c|c|c|c|}
\hline CUESTIONARIOS & EDAD & MEDIA & $\mathrm{F}$ & $p$ \\
\hline \multirow{5}{*}{ INTERNET } & $18-20$ & 0,9479 & \multirow{5}{*}{1,981} & \multirow{5}{*}{0,079} \\
\hline & $21-23$ & 0,7939 & & \\
\hline & $24-26$ & 0,4862 & & \\
\hline & $27-29$ & 0,9615 & & \\
\hline & $>29$ & 1,1304 & & \\
\hline \multirow{5}{*}{ JUEGO } & $18-20$ & 0,0674 & \multirow{5}{*}{2,997} & \multirow{5}{*}{0,011} \\
\hline & $21-23$ & 0,1333 & & \\
\hline & $24-26$ & 0,1101 & & \\
\hline & $27-29$ & 0,0400 & & \\
\hline & $>29$ & 0,3182 & & \\
\hline \multirow{5}{*}{ COMPRAS } & $18-20$ & 0,7638 & \multirow{5}{*}{3,957} & \multirow{5}{*}{0,001} \\
\hline & $21-23$ & 0,6258 & & \\
\hline & $24-26$ & 0,3784 & & \\
\hline & $27-29$ & 0,4800 & & \\
\hline & $>29$ & 0,7826 & & \\
\hline
\end{tabular}

En cuanto al uso del teléfono móvil, parece que los más jóvenes son los que más horas pasan al día hablando por teléfono. Concretamente, pasan una hora al dia hablando por el móvil, un 49,4\% (159) de 18-20 años, un 32,9\% (106) de 21-23 años, un 11,2\% (36) de 24-26 años, un 4,7\% (15) y un $1,9 \%(6)$ mayores de 29 años $(p<0,05)$. No hay diferencias de edad en cuanto al número de mensajes de texto enviados al día ni en la pregunta "¿Has intentado sin éxito reducir el dinero que gastas en móvil?".

\section{Diferencias en función del curso:}

Relacionando el curso de los estudiantes con la media obtenida en cada uno de los cuestionarios, se puede decir que sólo se dan diferencias significativas en el cuestionario de Adicción a las Compras con una $F(3,985)=5,075 ; p<0,05$. No se dan diferencias en el cuestionario de Adicción a Internet ni en el de Adicción al Juego. Obtienen una mayor puntuación media en el cuestionario, los alumnos de primer y segundo curso.

Tabla 3. Análisis de Varianza entre la variable del curso y el Test de adicción a las compras, el Test de adicción a Internet (Echeburúa, 2003) y el Cuestionario Breve de Juego patológico (Fernández-Montalvo y Echeburúa, 1997).

\begin{tabular}{|c|c|c|c|c|}
\hline CUESTIONARIOS & CURSO & MEDIA & $\mathrm{F}$ & $p$ \\
\hline \multirow{4}{*}{ INTERNET } & Primero & 0,9011 & \multirow{4}{*}{2,415} & \multirow{4}{*}{0,065} \\
\hline & Segundo & 1,0415 & & \\
\hline & Tercero & 0,7636 & & \\
\hline & Cuarto & 0,5934 & & \\
\hline \multirow{4}{*}{ JUEGO } & Primero & 0,0765 & \multirow{4}{*}{0,693} & \multirow{4}{*}{0,556} \\
\hline & Segundo & 0,1176 & & \\
\hline & Tercero & 0,1079 & & \\
\hline & Cuarto & 0,0899 & & \\
\hline \multirow{4}{*}{ COMPRAS } & Primero & 0,7318 & \multirow{4}{*}{5,075} & \multirow{4}{*}{0,002} \\
\hline & Segundo & 0,8018 & & \\
\hline & Tercero & 0,5190 & & \\
\hline & Cuarto & 0,6344 & & \\
\hline
\end{tabular}

En cuanto al uso del teléfono móvil y el curso al que pertenecen los universitarios, reconocen hablar dos horas al día por el teléfono móvil el 10,4\% (33) de los alumnos de primero, el 10,9\% (21) de los alumnos de segundo, el $7,3 \%(22)$ de los alumnos de tercero y el 8,7\% (8) de los alumnos de cuarto $(p<0,05)$. Un 18,1\% (65) de los alumnos de primero reconocen mandar de 6 a 10 mensajes SMS al día, un 19,6\% (43) los alumnos de segundo, un 16,3\% (52) los alumnos de tercero y un 9,7\% (9) los alumnos de cuarto $(p<.05)$. En general, parece que los alumnos de los cursos inferiores son los que hacen un mayor uso del teléfono móvil.

\section{Diferencias en función de la macroárea de cono- cimiento (ciencias o letras)}

Teniendo en cuenta las puntuaciones medias en cada uno de los cuestionarios en función de la variable área de conocimiento (letras o ciencias), según el Análisis de Varianza sólo se dan diferencias estadísticamente significativas en el cuestionario de Adicción a las Compras con una $F(1,987)=$ 19,158; $p<0,05$. Los alumnos de la macroárea de letras son los que obtienen una mayor puntuación en el cuestionario. No se dan diferencias significativas en los cuestionarios de Adicción a Internet y de Adicción al Juego. 
Tabla 4. Análisis de Varianza entre la variable macroárea de conocimiento y, el Test de adicción a las compras, el Test de adicción a Internet (Echeburúa, 2003) y el Cuestionario Breve de Juego patológico (Fernández-Montalvo y Echeburúa, 1997).

\begin{tabular}{|l|c|c|c|c|}
\hline CUESTIONARIOS & MACROÁREA & MEDIA & $\mathrm{F}$ & $\mathrm{p}$ \\
\hline \multirow{3}{*}{ INTERNET } & Letras & 0,8535 & 0,010 & 0,921 \\
\cline { 2 - 3 } & Ciencias & 0,8636 & & \\
\hline \multirow{2}{*}{ JUEGO } & Letras & 0,0702 & \multirow{2}{*}{3,588} & 0 \\
\cline { 2 - 3 } & Ciencias & 0,059 \\
\hline \multirow{2}{*}{ COMPRAS } & Letras & 0,8229 & \multirow{2}{*}{19,158} & \multirow{2}{*}{0,001} \\
\cline { 2 - 3 } & Ciencias & 0,5663 & & \\
\hline
\end{tabular}

En cuanto al uso del teléfono móvil, los alumnos de letras reconocen pasar entre dos horas al día hablando por teléfono con un $12,8 \%$ (45) y el grupo de ciencias con un $7,1 \%$ (39) $(p<0,05)$. En cuanto a los mensajes de texto, un 20\% (80) de los alumnos de letras mandan de 6 a 10 mensajes de texto al día frente al 15,1\% (89) de alumnos de ciencias $(p<.05)$, pero cuando la frecuencia de los mensajes aumenta (más de 10) son los alumnos de letras los que mayor frecuencia presentan 10,2\% (41) frente al 5,8\% (34) $(p<.05)$.

Para ver qué tipo de función ejercen variables como la edad, el sexo, el curso, etc. en las puntuaciones de los diferentes cuestionarios, se realizó un Análisis de Regresión Logística Binaria por pasos hacia adelante. Las variables dependientes fueron las puntuaciones de los cuestionarios (adicción a las compras, al juego y a Internet), donde una puntuación 0 era bajo riesgo a tener un problema de adicción (de 0 a 1 items con respuesta afirmativa) y 1 era una alta probabilidad de tener un problema de adicción a las compras o al juego (dos o más respuestas afirmativas). En el caso del cuestionario de adicción a Internet, la puntuación 0 agrupaba a los sujetos que no tenían riesgo de tener adicción a Internet (de 0 a 3 respuestas afirmativas) y 1 agrupaba a los jóvenes que sí estaban en riesgo de tener un posible problema de adicción a Internet (de 4 a 9 respuestas afirmativas).

Concretamente, para el cuestionario de adicción a Internet, la variable que entra a formar parte del modelo es la variable sexo. Una Odds Ratio inferior a 1 (situándose también los límites de consistencia interna por debajo de 1), teniendo en cuenta que 1 es ser hombre y 2 es ser mujer, indica que ser mujer $(O R=0,313$; IC 95\%=0,169-0,582) es un factor protector a la hora de tener una posible adicción a navegar por la red.

En cuanto al cuestionario de adicción al juego, las variables que entran a formar parte del modelo son el sexo y la macroárea de conocimiento (ciencias o letras). Para la macroárea de conocimiento, según la $\mathrm{OR}=8,034$; IC 95\%= 0,939-68,709; pertenecer a la macroárea de ciencias es un factor de riesgo a la hora de presentar una adicción al juego y para la variable sexo, según la $O R=0,185$; IC $95 \%=0,040-$ 0,853 ; ser mujer es un factor protector ante una posible adicción al juego.

Para el cuestionario de adicción a las compras, las variables que entran a formar parte del modelo son la edad y la macroárea de conocimiento (ciencias o letras). Una
Odds Ratio inferior a 1 (situándose también los límites de consistencia interna por debajo de 1), indica que ser de la macroárea de ciencias $(O R=0,594$; IC 95\%=0,419-0,843;) y tener más edad $(O R=0,791$; IC $95 \%=0,640-0,978)$ son factores protectores ante la posibilidad de presentar una adicción a las compras.

Tabla 5. Análisis de regresión logística entre la puntuación obtenida en cada uno de los cuestionarios (adicción a Internet, las compras y el juego) y variables como la edad, el sexo, el curso y la macroárea de conocimiento.

\begin{tabular}{|l|c|c|c|c|c|c|}
\hline & B & E.T. & Wald & p & OR & IC 95\% \\
\hline Adicción a Internet & $-1,161$ & 0,316 & 13,506 & 0,001 & 0,313 & $0,169-0,582$ \\
\hline Sexo & 2,084 & 1,095 & 3,621 & 0,057 & 8,034 & $0,939-68,709$ \\
\hline Adicción al Juego & 1,690 & 0,781 & 4,683 & 0,030 & 0,185 & $0,040-0,853$ \\
\hline Macroárea & 0,452 & 0,184 & 6,041 & 0,014 & 0,636 & $0,419-0,843$ \\
\hline Sexo & 0,222 & 0,108 & 4,221 & 0,040 & 0,801 & $0,640-0,978$ \\
\hline Adicción a las Compras \\
\hline Macroárea
\end{tabular}

NOTA: $B=$ coeficiente; $E . T=$ error estándar; $p=$ probabilidad; $O R=$ odds ratio; $I . C .95 \%=$ intervalo de confianza 95\%.

\section{DISCUSIÓN}

Con este trabajo de investigación se pretendía analizar los patrones del uso y/o abuso de comportamientos susceptibles de una posible adicción como el juego, el uso de Internet, el uso del teléfono móvil y las compras en estudiantes universitarios de Córdoba y observar la relación que existía entre variables como la edad, el sexo, el curso, y las macro áreas de conocimiento. La problemática de las adicciones no se puede limitar exclusivamente al consumo de sustancias psicoactivas, sino que existen hábitos de conducta aparentemente no perjudiciales, que pueden acarrear una serie de consecuencias negativas, cercano a lo que se considera por adicción a sustancias psicoactivas en jóvenes. En general, parece que los estudiantes de la Universidad de Córdoba no presentan una alta prevalencia en adicciones conductuales del tipo navegar por Internet, juego, compras y uso del teléfono móvil, coincidiendo con otros trabajos de investigación (Becoña, et al., 2001; García, et al., 2008).

Los universitarios utilizan Internet y reconocen extraer un gran placer social de la red. En este sentido, se confirma el dato de otras investigaciones donde el uso de Internet por sí solo puede que no sea la razón fundamental del abuso, sino su capacidad de "recompensa" proporcionada a través del ocio o las relaciones internautas, lo que podría producir cierta dependencia (García, et al., 2008; Luengo, 2004; Madrid, 2000; Muñoz-Rivas, et al., 2003; SánchezCarbonell, et al., 2008). Mediante Internet y el móvil los jóvenes se convierten en elementos activos que ejercitan una conducta gratificante $y$, como tal, susceptible de adicción (Sánchez-Carbonell et al., 2008); recompensa, que no sólo encuentran en las relaciones sociales dirigidas a través de la red y que podrían ser explicadas por el cambio 
de hábitos y comportamientos sociales, sino que además utilizan este recurso para adquirir objetos o archivos que les resultan irresistibles. Así, relacionando la puntuación del cuestionario sobre Adicción a Internet (Echeburúa, 2003) con variables como la edad, el sexo, el curso o la macroárea de conocimiento, se puede decir que se dan diferencias entre hombres y mujeres, destacando los hombres con una mayor puntuación. Esto coincide con los datos ofrecidos en otros trabajo de investigación (Muñoz-Rivas, et al., 2003). No hay diferencias entre los estudiantes en función de la edad, del curso, ni de las macroáreas de conocimiento. La Regresión Logística realizada identifica como factor protector ser mujer. Es decir, que las jóvenes universitarias tienen menor riesgo de presentar una adicción a Internet. Este dato confirma el uso diferencial de los recursos de la red entre hombres y mujeres, confirmando la evidencia observada desde hace años en cuanto al interés que despiertan las TIC's entre los varones (Muñoz-Rivas, et al., 2003).

El juego, que hasta hace algunos años era una actividad básicamente realizada por adultos (Secades y Villa, 1998), se ha convertido en una conducta placentera que despierta el interés de los jóvenes, iniciándose en el juego de las máquinas tragaperras por entretenimiento y motivados por el dinero (Atienza, 2006). Además, con el uso de las TIC's, la adición al juego ya no es exclusivamente aplicable a las máquinas tragaperras, sino que los videojuegos y el juego on-line son comportamientos también susceptibles de generar adicción (Echeburúa y De Corral, 2008; Salinas y Roa, 2002; Tejeiro, 2001). En este trabajo, y teniendo en cuenta el Cuestionario Breve de Juego Patológico utilizado (Fernández-Montalvo y Echeburúa, 1997), se puede decir que se dan diferencias significativas entre hombres y mujeres, obteniendo los hombres una mayor puntuación; también se dan diferencias entre los rangos de edad, donde los mayores de 29 años presentan una mayor puntuación, seguidos del rango 21-23, 24-26 y 18-20. Parece que los más jóvenes son los que menos riesgo de adicción a jugar tienen. No se dan diferencias entre los cursos ni entre las macroáreas de conocimiento. Teniendo en cuenta los resultados de la Regresión Logística, parece que pertenecer a una carrera de ciencias es un factor de riesgo, es decir, puede tener una mayor probabilidad de tener un problema de adicción al juego; y por otro lado, ser mujer resulta ser un factor protector. El interés por el juego es diferente entre géneros. La inclusión de las mujeres en el mundo del juego y de los juegos electrónicos no sigue el mismo ritmo ni intensidad que la de los hombres (Castellana, et al., 2007) y por lo tanto, la posibilidad de presentar una posible adicción parece ser menor.

Con respecto al cuestionario utilizado para valorar una posible adicción a las compras, cabe destacar que existen diferencias entre hombres y mujeres, destacando en esta ocasión las mujeres. También se dan diferencias entre los grupos de edades, siendo los más jóvenes los que obtienen una puntuación mayor en el cuestionario; entre los diferentes cursos, destacan los de primero y segundo; en cuanto a las diferencias entre las macroáreas de conocimiento, destacan los alumnos de las carreras de letras por presentar mayor riesgo a tener un problema de adicción a las compras.
Atendiendo a la Regresión Logística realizada, se puede decir que la variable edad y la variable macroárea de conocimiento actúan como factores protectores, es decir, a mayor edad y el hecho de pertenecer a una carrera de ciencias, aumenta la probabilidad de no presentar un problema de adicción a las compras. Con lo cual, ser mujer y estar cursando una carrera de letras pueden ser factores de riesgo a tener en cuenta.

En cuanto al uso del móvil, los resultados parecen señalar que son las mujeres las que mandan un mayor número de mensajes, las que reconocen fracasar a la hora de reducir el gasto del móvil y las que pasan más horas hablando por él. Se confirma el interés que despierta este tipo de tecnologías en las mujeres en detrimento de otras (Castellana, et al., 2007).

En conclusión, se puede decir que las prevalencias mostradas en el presente estudio, indican que los jóvenes universitarios conviven con las TIC's y presentan un uso excesivo de comportamientos cercanos a ser valorados como una adicción tanto a las compras, al juego, al móvil y/o al uso de Internet. Ante esta situación y para el diseño y la realización de futuros programas de prevención (Cuesta y Menéndez, 2009), es importante hacer hincapié en la toma de decisiones de estos jóvenes universitarios, ya que una opción apropiada, ante un posible uso o abuso de cualquiera de los anteriores comportamientos, puede evitar una problemática posterior o una dependencia futura a las mismas. La importancia de conocer y analizar estos fenómenos incide en el avance de modelos o propuestas de intervención que mejoren las posibles patologías derivadas de éstos.

Como limitaciones determinadas por el propio diseño del estudio, destaca la recogida de datos en el propio aula, que si bien es el entorno más accesible, también conlleva un cierto sesgo de selección que conlleva una infraestimación en los resultados, ya que los alumnos que van a clase con regularidad son los que tienen, en general, hábitos de vida más saludables (Mas, Nerín, Barrueco, Cordero, Guillén, Jiménez-Ruiz, et al., 2004); el sesgo de deseabilidad social inherente al uso de autoinformes, aunque es de suponer que no será muy acusado ya que se garantizó a los participantes su anonimato (Font-Mayolas, Grass y Planes, 2006); y la dificultad para generalizar los resultados, ya que la muestra procede de un único centro universitario español. Además, es necesario señalar que a pesar del interés que suscitan los resultados de la Regresión Logística, es necesario ser cauto a la hora de generalizar dichos resultados, ya que el tamaño de la muestra de alumnos en riesgo es pequeña y no son clasificados correctamente.

En este sentido, para futuras líneas de investigación, sería interesante replicar este tipo de trabajos con instrumentos de evaluación más adecuados que detecten aquella población susceptible de estar en riesgo; ampliar estudios que consideren los aspectos cognitivos que subyacen a estas conductas de cara a planificar actuaciones con estos estudiantes; y profundizar en las variables de protección y de riesgo que están interviniendo en la adicción de comportamientos relacionados con las compras, el juego, el móvil e Internet. 


\section{AGRADECIMIENTOS}

Este trabajo ha sido posible gracias al convenio de colaboración entre la Consejería para la Igualdad y Bienestar Social de la Junta de Andalucia, y la Universidad de Córdoba en materia de drogodependencias y adicciones.

\section{REFERENCIAS}

Adès, J. y Lejoyeux, M. (2003). Las nuevas adicciones: Internet, sexo, deporte, compras, trabajo, dinero. Barcelona: Kairós.

Alonso-Fernández, F. (2003). Las nuevas adicciones. Madrid: TEA.

Arbinaga, F. (1996a). Conductas de juego con apuestas y uso de drogas en una muestra de adolescentes de la ciudad de Huelva. Análisis y Modificación de Conducta, 22, 577-601.

Arbinaga, F. (1996b). El juego patológico en estudiantes menores de 18 años: incidencia, uso de drogas y variables asociadas. Adicciones, 8,331-348.

Arbinaga, F. (2000). Estudio descriptivo sobre el juego patológico en estudiantes (8-17 años): caracteristicas sociodemográficas, consumo de drogas y depresión. Adicciones, 12, 493-505.

Arenas, F.J., Carmona, J., Rodríguez, A. y Tatnell, R. (2002). Patrones de consumo de drogas en la Universidad de Córdoba. Córdoba: Patronato Provincial de Servicios Sociales de Córdoba.

Atienza, J. (2006). Cuando jugar no es divertido: Una guía para conocer el juego patológico. Servicio Provincial de Drogodependencias: Diputación de Cádiz.

Becoña, E. (1993). El juego compulsivo en la Comunidad Autónoma Gallega. Santiago de Compostela: Consellería de Sanidade da Xunta de Galicia.

Becoña, E. (1996). La ludopatía. Madrid: Aguilar

Becoña, E. (2003). Bases científicas de los programas de prevención de las drogodependencias. Madrid: Plan Nacional sobre Drogas.

Becoña, E., Miguez, Ma. C. y Vázquez, F.L. (2001). El juego problema en los estudiantes de Enseñanza Secundaría. Psicothema, 13, 551556.

Bianchi, A. y Phillips, J.G. (2005). Psychological predictors of problem mobile phone use. Cyberpsychology \& Behavior, 8, 39-51.

Castellana, M., Sánchez-Carbonell, X., Graner, C. y Beranuy, M. (2007). El adolescente ante las tecnologias de la información y la comunicación: internet, móvil y videojuegos. Papeles del Psicológo, 28, 196-204.

Criado, M.A. (2005). Enfermos del móvil. Recuperado el 10 de julio de 2009 de: http//www.el-mundo.es/ariadna/2005/218/1106327395. html

Cuesta, U. y Menéndez, T. (2009). Prevención, comunicación y nuevas tecnologias: Aspectos psicológicos entre jóvenes universitarios. En J del Pozo, L. Pérez y M. Ferreras (Eds.), Adicciones y nuevas tecnologías de la información y de la comunicación. Perspectivas de su uso para la prevención y el tratamiento. La Rioja: Consejería de Salud.

Dimmick, J.W., Kline, S. y Strafford, L. (1994). The gratification niches of personal E-mail and the telephone. competition, displacement, and complementarily. Communication Research, 27, 227-248.

Echeburúa, E. (1999). ¿Adicciones sin drogas?. Bilbao: Desclée de Brouwer.
Echeburúa, E. (2003). ¿Adicciones sin drogas? Las nuevas adicciones: juego, sexo, comida, compras, trabajo, Internet. Editorial Bilbao: Desclée de Brouwer.

Echeburúa, E. y De Corral, P. (2008). Juego responsable: ¿una alternativa para la prevención y el tratamiento de la ludopatía? Adicciones, 20.321-326.

Fernández-Montalvo, J., Echeburúa, E.y Báez, C. (1997). El cuestionario breve de juego patológico (CBJP): un nuevo instrumento de "screening", Análisis y Modificación de Conducta, 21, 76, 211 225.

Font-Mayolas, S., Grass, M.E. y Planes, M. (2006). Análisis del patrón de consumo de cannabis en estudiantes universitarios. Adicciones, 18, 337-344

García del Castillo, J. A., Terol, M. C., Nieto, M., Lledó, A., Sánchez, S., Martín-Aragón, M. y Sitges, E. (2008). Uso y abuso de Internet en jóvenes universitarios. Adicciones, 20, 131-142.

González Duro, E. (2005). El riesgo de vivir. Madrid: Temas de Hoy.

Holden, C. (2001). Behavioral addictions: do they exist? Science, 294 980-982.

Hölfich, J. y Rössler, P. (2002). Más que un teléfono: El teléfono móvil y el uso del SMS por parte de los adolescentes alemanes. Resultados de un estudio piloto. Estudios de Juventud, 57, 79-99.

Kamibeppu, K. y Sugiura, H. (2005). Impact of the mobile phone on junior high-school students' friendships in the Tokio metropolitan area. Cyberpsychology \& Behaviour, 8, 121-130.

Lemon, J. (2002). Can we call behaviours addictive?. Clinical psychologist, 3, 44-49.

Lesieur, H.R. y Rosenthal, R.J. (1991). Pathological gambling: A review of the literature (Prepared for the American Psychiatric Association Task Force on DSM-IV Committee on Disorders of Impulse Control Not Elsewhere Classified). Journal of Gambling Studies, 7, 5-40.

Leung, L. y Wei, R. (2000). More than just talk on the move: Uses and gratifications of the cellular phone. Journalism \& Mass Communication Quarterly, 77, 308-320.

Luengo, A. (2004). Adicción a Internet: Conceptualización y propuesta de intervención. Revista Profesional Española de Terapia Cognitivo-Conductual, 2, 22-52.

Madrid, R.I. (2000). La adicción a internet. Psicología Online. Recuperado a 9 de julio de 2009, de http://www.psicologia-online.com/ colaboradores/nacho/ainternet.htm

Marlatt, G. A. y Gordon, J.R. (Ed.) (1985). Relapse prevention: Maintenance strategies in the treatment of addictive behaviors. New York: Guilford Press.

Mas, A., Nerín, I., Barrueco, M., Cordero, J. Guillén, D., Jiménez-Ruiz, C. et al., (2004). Consumo de tabaco en estudiantes de sexto curso de medicina de España. Archivos de Bronconeumonología, 40, 403-408.

Montero, I. y León, O. (2007). A guide for naming research studies in Psychology. International Journal of Clinical and Health Psychology, 7, 847-862.

Muñoz-Rivas, M. J. y Agustín, S. (2005). La adicción al teléfono móvil. Psicología Conductual, 13, 481-493.

Muñoz-Rivas, M.J., Navarro Perales E. y Ortega de Pablo, N. (2003). Patrones de uso de Internet en población universitaria española. Adicciones, 15, 137-144 
O'Keefe, G. J. y Sulanowski, B. K. (1995). More than just talk: Uses, gratifications, and the telephone. Journalism \& Mass Communication Quarterly, 72, 922-933.

Protégeles (2005). Seguridad infantil y costumbres de los menores en el empleo de la telefonía móvil. Madrid: Protégeles y Defensor del Menor, Madrid, 2005. Disponible en [fecha de consulta 20/06/09]: http://www.dmenormad. es/pdf/ estudiotelefonosmoviles.doc.

Salinas, J,M, y Roa J.M. (2002). El screening de la adicción al juego mediante internet. Adicciones, 14, 303-312.

Sánchez-Carbonell, X., Beranuy, M., Castellana, M., Chamarro, A. y Oberst, U. (2008). La adicción a Internet y al móvil: ¿moda o trastorno? Adicciones, 20, 149-160.
Secades, R. y Villa, A. (1998). El juego patológico. Prevención, evaluación y tratamiento en la adolescencia. Madrid: Pirámide.

Tejeiro, R. (2001). La adicción a los videojuegos. Una revisión. Adicciones, 13, 407-413.

Viñas, F., Juan, J., Villar, E., Caparros, B., Perez, I. y Cornella, M. (2002). Internet y psicopatologia: las nuevas formas de comunicación y su relación con diferentes indices de psicopatología. Clínica y Salud, 13, 235-256.

Young, K.S. (1996). Psychology of computer use: XL. Addictive use of the internet: A case that breaks the stereotype. Psychological Reports, 79, 899-902. 
